\title{
The Miscibility of Homopolymer/Random Copolymer Blends I. Poly(styrene-co-acrylonitrile)/Poly(ethyl methacrylate) and Poly(styrene-co-acrylonitrile)/Poly(methyl methacrylate) Blends
}

\author{
Hanqiao Feng, ${ }^{\dagger}$ Chaohui Ye, and Zhiliu FenG* \\ Laboratory of Magnetic Resonance and Atomic and Molecular Physics, Wuhan Institute of Physics, The Chinese Academy of Sciences, \\ Wuhan 430071, People's Republic of China \\ * Polymer Physics Laboratory, Changchun Institute of Applied Chemistry, The Chinese Academy of Sciences. \\ Changchun 130022, People's Republic of China
}

(Received November 27, 1995)

\begin{abstract}
The miscibility of blends of poly(styrene-co-acrylonitrile) (SAN) with poly(methyl methacrylate) (PMMA) or poly(ethyl methacrylate) (PEMA) has been investigated by means of NMR and DSC techniques. It is found that there are intermolecular interactions between the phenyl groups in SAN and carbonyl groups in PMMA or PEMA, and the strength of this intermolecular interaction strongly depends on the properties of ester side groups in PEMA or PMMA, composition of the blends and a certain composition of the copolymer. It is this specific interaction instead of the intramolecular repulsion force within the copolymer that plays a key role for the miscibility of SAN/PMMA and SAN/PEMA blends.

KEY WORDS Miscibility $/{ }^{13} \mathrm{C}$ Cross Polarizational Magic Angle Spinning Nuclear Magnetic Resonance Differential Scanning Calorimetry /Intermolecular Interaction / Random Copolymer /
\end{abstract}

Recently extensive study on blends of random copolymer with homopolymer has been made ${ }^{1-16}$ because of their interesting phase behavior. A typical example of such blends is the blend of poly(styrene-co-acrylonitrile) (SAN) with poly(methyl methacrylate) (PMMA). These two polymers are known to be miscible when the copolymer's composition is within a particular range. However, neither components of the copolymer, i.e., polystyrene (PS) or polyacrylonitrile (PAN), is miscible with PMMA, which implies the absence of any specific interaction between one with another. Simple binary interaction models ${ }^{7,12-16}$ with various extension have been devoted to explain these effects through the concept of intramolecular repulsion force within the copolymers. Unfortunately, as pointed out in our recent paper, ${ }^{17}$ both its prerequisite and conclusion are not applicable to these blend systems because of oversimplification of the models. For example, some disagreements have been reported on studies of blends of PMMA with SAN with different contents of acrylonitrile (AN). Kwei et al. $^{3,9}$ studied in detail the miscibility of blends of PMMA with an SAN containing $28 \mathrm{wt} \%$ AN by various physical techniques, such as density, specific heat, dielectric relaxation, enthalpy relaxation, FT-IR and NMR. Their data indicate that the excess volume, enthalpy, and entropy of mixing for SAN-PMMA pair are extremely small in magnitude. The interaction between the two polymers is manifested by a downward shift of the infrared stretching frequency of the carbonyl group but only about 3\% of the PMMA segments contribute to the displaced peak. Furthermore, their NMR results suggest that there exists inhomogeneity on a characteristic length between the limits of 20 and $150 \AA$. However, based on the glass transition temperature $\left(T_{\mathrm{g}}\right)$ obtained and naked eye observation for the blends of PMMA with SAN containing a wide range $(0-33.0 \mathrm{wt} \%)$ of AN, Paul et al..$^{2,10,11}$ concluded that

\footnotetext{
$\dagger$ To whom correspondence should be addressed.
}

PMMA and SAN are miscible, when the AN content in SAN is in the range of 6.3 to $32.3 \mathrm{wt} \%$, and that the basis for the miscibility of SAN with PMMA seems to be that there exists a relatively strong repulsion force between styrene (St) and AN units within SAN copolymer. Aside from that their data are not precise enough, so far they have not obtained any direct experimental evidence for the existence of the repulsion interaction they assumed. It is noticed that in another paper Paul et al. made an ambiguous statement that the styrene-methacrylate interaction is weak and may be positive or negative depending on the structure of the methacrylate pendent group. ${ }^{8}$ In fact, so far there has been no one who can unambiguously explain the miscibility mechanism of SAN/PMMA blends. Therefore, it is of both scientifical and practical significance to investigate the mechanism of miscibility of PMMA with SAN containing a suitable AN content. NMR has been one of the most powerful tools for studying molecular motion, intermolecular interaction, and miscibility of polymer blends, ${ }^{18-20}$ and is possible to give the relevant information on a molecular level. In this series of studies, we will present the results of our study on the miscibility of several random copolymer and homopolymer pairs by NMR and other techniques such as DSC and FT-IR in order to understand the essence of the miscibility of such blends.

\section{EXPERIMENTAL}

The three polymers, SAN, PMMA, and PEMA, used in this study are all purchased from Polyscience Inc. (U.S.A.), and their $\eta$ or $M_{\mathrm{w}}$ and $T_{\mathrm{g}}$ 's are listed in Table I. Solution blending with tetrahydrofuran (THF) as solvent was used, and solution concentration was $10 \%(\mathrm{w} / \mathrm{v})$. Specimens with different compositions were cast from the solution at room temperature, and then dried under vacuum at $80^{\circ} \mathrm{C}$ for a week.

Solid state ${ }^{13} \mathrm{C}$ cross polarization/magic angle spin- 
ning (CPMAS) NMR experiments were performed on a BRUKER MSL-400 NMR spectrometer at $298.0 \mathrm{~K}$. The TOSS method was used for suppressing spinning side bands. The proton resonance frequency was 400.13 $\mathrm{MHz}$, and carbon resonance frequency was 100.63 $\mathrm{MHz}$, while $5.5 \mu \mathrm{s} 90^{\circ}$ pulse for ${ }^{13} \mathrm{C}$ nucleus and $4.8 \mu \mathrm{s}$ $90^{\circ}$ pulse for ${ }^{1} \mathrm{H}$ were used. Magic-angle spinning rate was $4.0 \mathrm{kHz} .{ }^{13} \mathrm{C}$ spin-lattice relaxation time were measured by $T_{1} \mathrm{CP}$ method, ${ }^{21}$ and ${ }^{1} \mathrm{H}$ spin-lattice relaxation times in the rotating frame, $T_{1 \rho}(\mathrm{H})$, were obtained from the ${ }^{13} \mathrm{C}$ CPMAS intensity with increasing contact times. ${ }^{22}{ }^{13} \mathrm{C}$ spectra were referred to the chemical shift of methyl group carbons of hexamethyl benzene which is $16.9 \mathrm{ppm}$.

A Perkin-Elmer DSC-2 apparatus was used to determine the glass transition temperatures $\left(T_{\mathrm{g}}\right)$ of the polymers and their blends. DSC curves of the specimens were recorded at a heating rate of $20 \mathrm{~K} \mathrm{~min}^{-1}$. The temperature at the midpoint of the heat capacity change of the DSC curve was taken as $T_{\mathrm{g}}$ of the specimen.

\section{RESULTS AND DISCUSSION}

\section{Miscibility of SAN/PEMA Blends}

Shown in Figure 1 are the ${ }^{13} \mathrm{C}$ CPMAS NMR spectra of SAN, PEMA and their blends with different compositions. The chemical shifts of some carbons and the relative resonance intensities of non-protonated and protonated aromatic carbons in Figure 1 are listed in Table II. The values of chemical shifts were automatically determined, they are not estimated by the lines with naked eye. The assignments of PEMA and SAN ${ }^{13} \mathrm{C}$ resonances are marked on the corresponding peaks in Figure 1.

From Figure 1 and Table II, it is obvious that addition of PEMA to SAN has marked influence on the electron density of the phenyl group of SAN, since the chemical shift of non-protonated aromatic carbon regularly shift down to lower field with increase in PEMA content, and the relative resonance intensity of the non-protonated aromatic carbon and protonated aromatic carbons increases with the increase in PEMA. At the same time, the chemical shifts of the other carbons in SAN shift towards the lower field in most cases. In addition the asymmetry of the non-protonated aromatic carbon peak becomes more and more obvious with increasing PEMA content. Very interestingly, it is noticed that just as mentioned in our previous paper, ${ }^{17}$ copolymerization of styrene with acrylonitrile results in a higher field shift of more than $3 \mathrm{ppm}$ of the non-protonated aromatic carbon, a relatively less lower field shift of the protonated (aromatic and aliphatic) carbons in the styrene unit, as compared with the chemical shifts of the homopolystyrene. At the same time, copolymerization has brought forth a higher field shift more than $3 \mathrm{ppm}$ of the aliphatic carbons in acrylonitrile unit as compared with that of homopolyacrylonitrile. The lower field shifts of the protonated carbons in styrene and the higher field shift of the aliphatic carbons are the results of the electron transition from styrene unit to acrylonitrile unit, due to the formation of charge transfer complexes during copolymerization. A possible explanation for the unusually higher field shift of the non-protonated aro-
Table I. Physical properties of the polymers used in this study

\begin{tabular}{lccc}
\hline Polymer & $\eta$ & $M_{w} \times 10^{-4}$ & $T_{\mathrm{g}} / \mathrm{K}$ \\
\hline SAN & & - & 368 \\
PMMA & 0.43 & - & 372 \\
PEMA & - & 7.8 & 340
\end{tabular}

a The AN content in SAN is $25 \%$ by weight.

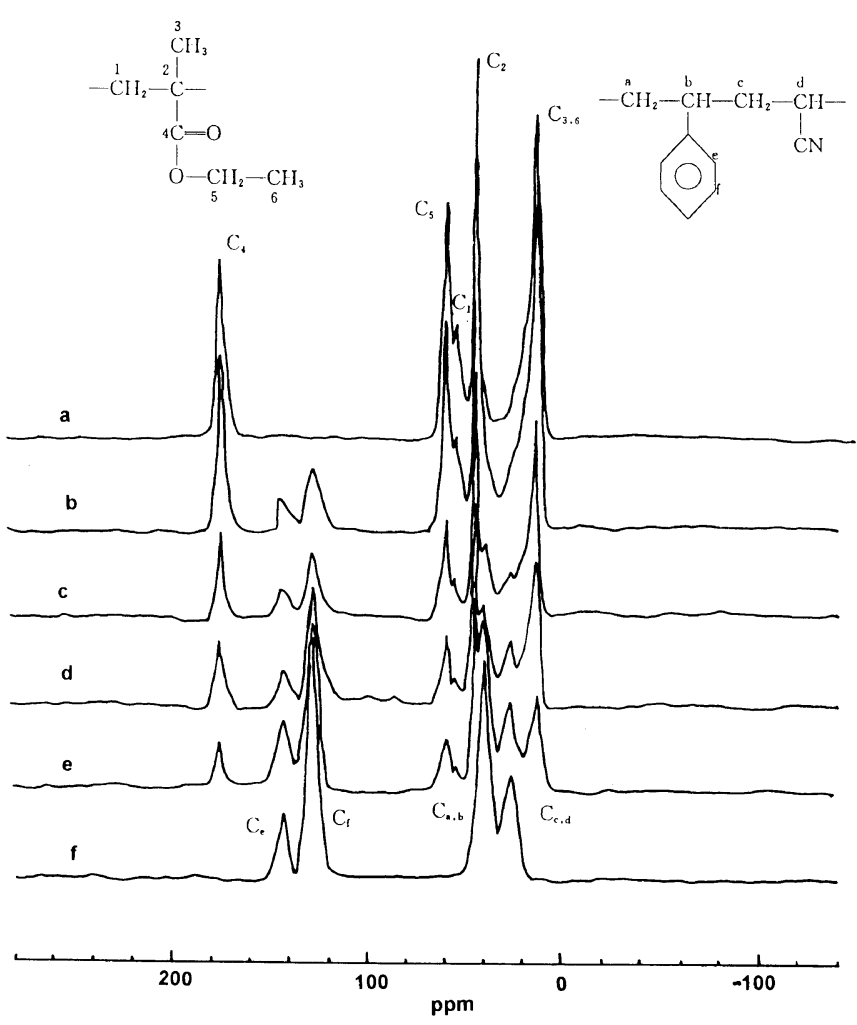

Figure 1. ${ }^{13} \mathrm{C}$ CPMAS NMR spectra of SAN, PEMA, and their blends, SAN/PEMA: a) $0: 100$; b) $20: 80$; c) $40: 60$; d) $60: 40$; e) $80: 20$; f) $100: 0$.

Table II. Chemical shifts of some carbons and the relative resonance intensity of non-protonated and protonated aromatic carbon in SAN, PEMA, and their blends

\begin{tabular}{cccccc}
\hline \multirow{2}{*}{$\begin{array}{c}\text { Composition } \\
\text { SAN/PEMA }\end{array}$} & \multicolumn{3}{c}{ Chemical shift/ppm } & & \multicolumn{2}{c}{ Relative intensity $I$} \\
\cline { 6 - 6 } \cline { 5 - 6 } & $\mathrm{C}=\mathrm{O}$ & Ar-C- & Ar-C-H & & $I(143.6 \mathrm{ppm}) / I(128.6 \mathrm{ppm})$ \\
\hline $100: 0$ & - & 143.6 & 128.6 & & 0.32 \\
$80: 20$ & 177.5 & 143.7 & 128.7 & & 0.34 \\
$60: 40$ & 177.5 & 144.1 & 129.0 & & 0.35 \\
$40: 60$ & 177.5 & 144.4 & 128.6 & & 0.50 \\
$20: 80$ & 177.4 & 144.7 & 129.0 & & 0.65 \\
$0: 100$ & 177.3 & - & - & & - \\
\hline
\end{tabular}

matic carbon may be that the field effect of nitrile group with electric quadrupole makes the electron density of the non-protonated aromatic carbon increase. Similar situation also happens to "alternating" copolymer poly(styrene-co-maleic anhydride), but does not to block copolymer poly(styrene- $b$-butadiene) because there are strong electron transfer interactions in the former, but not in the latter. It should be pointed out that such field effect strongly depends on the relative separation distance between and on the direction of the involved groups. ${ }^{23}$ 
An obvious change, which occurs due to the addition of PEMA to SAN, is the formation of electron transfer interactions between the phenyl groups and the carbonyl groups at the expense of the electron transfer interactions between the phenyl groups and the nitrile groups. As a result of the above change, the influence of the field effect of the nitrile group on the electron density of the nonprotonated aromatic carbon to some extent becomes weaker and weaker. In addition to the electron transfer from phenyl group to carbonyl group, the chemical shifts of the aromatic carbons regularly shift down to lower field as the PEMA content in the system is increased. It should be pointed out here that the existence of electron transfer interactions between the phenyl group and the carbonyl group does not mean that the interaction between the phenyl group and the nitrile group will disappear. In fact, the asymmetry of the peak at $143.6 \mathrm{ppm}$ strongly indicates the coexistence of the two interactions. As well known, the asymmetry of resonance peak comes from chemical shift dispersion, which originates from the resonance of the same atom or group with different chemical environments. In general, the increased electron density of the carbonyl group should lead to a high field shift. In fact, the chemical shift of the carbonyl group slightly moves down to the lower field. This is probably due to the formation of coplanar donor-acceptor structure, ${ }^{24}$ where aromatic deshielding effects prevail over the electron density change produced by electron transfer. Therefore, it may be concluded that there exist electron transfer interactions between PEMA and SAN, and it is this interaction that plays the key role in the miscibility of PEMA and SAN. The existence of such specific interaction is further supported by the spin-lattice relaxation times of the carbon atoms, $T_{1}(\mathrm{C})$, determined by NMR. As well known, $T_{1}(\mathrm{C})$ value can unambiguously describe the molecular motion of carbons. Therefore, determination of $T_{1}(\mathrm{C})$ value can be used to characterize the existence and the strength of intermolecular interaction. For example, very strong intermolecular interaction between PS and PPO leads to the phenyl rings of PS and those of PPO moving cooperatively, ${ }^{25}$ and interaction between PMMA and poly(vinyl phenol) (PVPh) results in the increased rate of motion of phenol group in $\mathrm{PVPh},{ }^{26}$ and the rate of motion of carbonyl group in PMMA decreasing. Mixing PVPh with poly(isobutyl methacrylate) has no effect on the molecular motion of both polymers due to the absence of intermolecular interaction between them.

The $T_{1}(\mathrm{C})$ values of PEMA, PMMA, SAN and two blends of SAN with these two polymethacrylates are listed in Table III. Obviously, mixing SAN with PEMA or PMMA results in a decreased $T_{1}(\mathrm{C})$ value of phenyl ring and an increased $T_{1}(\mathrm{C})$ value of carbonyl group, i.e., the rate of motion of phenyl group increases and the rate of motion of carbonyl group decreases due to mixing of them. This strongly supports our conclusion of the existence of intermolecular interaction between PEMA and SAN.

In addition, the level of homogeneity in SAN/PEMA systems was detected by determination of the proton spin-lattice relaxation times in the rotating frame $T_{1 \rho}(\mathrm{H})$. Figure 2 shows the plots of the logarithmic CPMAS

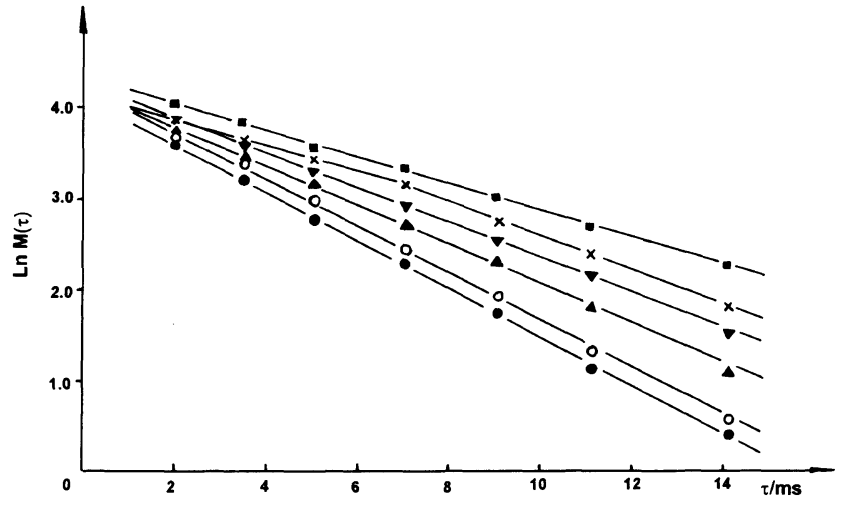

Figure 2. Plots of the logarithmic ${ }^{13} \mathrm{C}$ CPMAS resonance intensity $v s$. time of proton spin-locking of SAN/PEMA blends, SAN/PEMA:

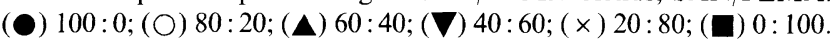

Table III. $T_{1}(\mathrm{C})$ values of SAN, PEMA, PMMA, and blends of SAN/PMMA $(60: 40)$ and SAN/PEMA $(60: 40)$

\begin{tabular}{lcc}
\hline \multirow{2}{*}{ Specimen } & \multicolumn{2}{c}{$T_{1}(\mathrm{C}) / \mathrm{s}$} \\
\cline { 2 - 3 } & $\mathrm{C}=\mathrm{O}$ & $\mathrm{Ar}-\mathrm{C}-\mathrm{H}$ \\
\hline SAN & - & 33.5 \\
PMMA & 16.3 & - \\
PEMA & 19.7 & - \\
SAN/PMMA & 23.2 & 27.0 \\
SAN/PEMA & 20.3 & 27.8 \\
\hline
\end{tabular}

${ }^{13} \mathrm{C}$ resonance intensity $v s$. time of proton spin-locking, from which, it is clearly seen that the decays of the magnetizations are exponential except for the one with SAN/PEMA $(20: 80)$ by weight. Their $T_{1 \rho}(\mathrm{H})$ values fitted by computer are listed in Table IV. The exponential decays of the proton relaxation indicate that SAN/PEMA blends are mixed intimately, in which spin diffusion via dipole-dipole interaction is effective enough to generate a single average $T_{1 \rho}(\mathrm{H})$ value. However, inhomogeneity exists in blends with SAN/PEMA $(20: 80)$ by weight, as it shows two $T_{1 \rho}(\mathrm{H})$ values. The results of NMR are in good agreement with those of DSC measurements for the same specimens (see Table IV).

It is very surprising to find that the $T_{\mathrm{g}}$ value of SAN/ PEMA $(80: 20)$ blend is higher than those of both the component polymers, and that the lower one of the two $T_{\mathrm{g}}$ values in SAN/PEMA $(20: 80)$ system is lower than that of pure PEMA. In order to confirm the reliability of the $T_{\mathrm{g}}$ values, the DSC measurement was repeated, the results are within experimental error. A reasonable explanation for the increase in $T_{\mathrm{g}}$ value is that the formation of charge transfer complex reduces the free volume of the system and hinders the molecular motion. For the partially miscible blend (SAN/PEMA, 20:80), the low $T_{\mathrm{g}}$ even lower than that of pure PEMA may be ascribed to that an enlarged free volume occurs due to mixing SAN with PEMA, but its mechanism is unknown and needs further investigation.

\section{Miscibility of SAN/PMMA Blends}

For comparison, the miscibility of SAN/PMMA blends was also studied by means of NMR and DSC. It is difficult from the results of DSC to give any informa- 


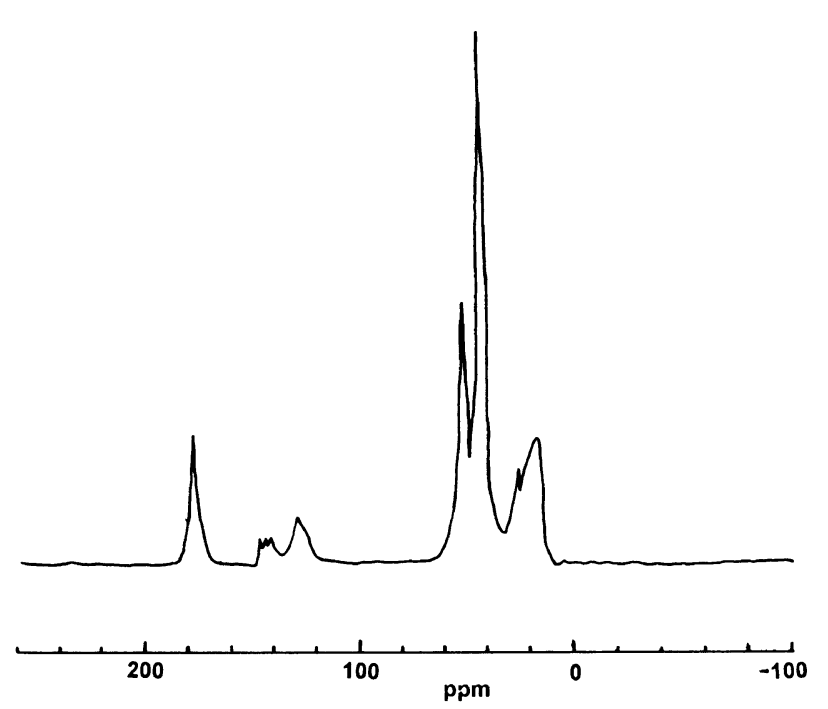

Figure 3. ${ }^{13} \mathrm{C}$ CPMAS NMR spectrum of SAN/PMMA $(20: 80)$ blend.

Table IV. $T_{1 \rho}(\mathrm{H})^{\mathrm{a}}$ and $T_{\mathrm{g}}$ values of SAN, PEMA, and their blends

\begin{tabular}{ccc}
\hline SAN/PMMA & $T_{1 \rho}(\mathrm{H}) / \mathrm{ms}$ & $T_{\mathrm{g}} / \mathrm{K}$ \\
\hline $100: 0$ & 8.9 & 368 \\
$80: 20$ & 8.6 & 372 \\
$60: 40$ & 9.3 & 365 \\
$40: 60$ & 12.2 & 362 \\
$20: 80$ & $10.2,16.6$ & 320,358 \\
$0: 100$ & 13.0 & 340
\end{tabular}

${ }^{\text {a }}$ Estimated error $< \pm 5 \%$.

tion about the miscibility SAN/PMMA blends because the $T_{\mathrm{g}}$ 's of SAN and PMMA are so close that it is hard to distinguish them. Fortunately, NMR can unambiguously give information about the miscibility of SAN/ PMMA blends, which is slightly different from that of SAN/PEMA according to their $T_{1 \rho}(\mathrm{H})$ values. When $\mathrm{SAN}$ is $80 \mathrm{wt} \%$ in blend, the decay of proton magnetizations is exponential for SAN/PEMA $(80: 20)$ blend, but is nonexponential for SAN/PMMA $(80: 20)$ system. On the contrary, a single $T_{1 \rho}(\mathrm{H})$ value occurs for SAN/ PMMA $(20: 80)$ blend, and two $T_{1 \rho}(\mathrm{H})$ values appear for SAN/PEMA $(20: 80)$ blend. One thing in common for SAN/PMMA and SAN/PEMA blends is that there are intermolecular interactions between phenyl groups and carbonyl groups. It is this specific interaction which makes SAN miscible with PMMA or PEMA. Besides, the lower field shift, widening and splitting of the nonprotonated aromatic carbon resonance peak of SAN/ PMMA $(20: 80)$ are more obvious than those of SAN/ PEMA (20:80) (see Figure 3). This suggests that the intermolecular interaction between PMMA and SAN is stronger than that between PEMA and SAN, which is in good agreement with that the cloud point temperature of SAN/PMMA system is higher than that of SAN/ PEMA. ${ }^{10}$

From the above discussion, the following conclusions may be drawn: it is the intermolecular charge transfer interaction between SAN and PMMA or PEMA instead of intramolecular repulsive force within SAN copolymer, that brings about miscibility between SAN and PMMA or PEMA. And the strength of the intermolecular interactions obviously depends on the properties of the pendent group of polymethacrylates, the composition of the blends and a defined composition of the copolymer as predicted by our proposed theory. ${ }^{17}$ Therefore, the miscibility of SAN/PEMA blends is slightly different from that SAN/PMMA blends.

Acknowledgments. The authors are grateful to the financial support granted by The National Key Projects for Fundamental Research "Macromolecular Condensed State," the State Science and Technology Commission of China and the Laboratory of Magnetic Resonance and Atomic and Molecular Physics, Wuhan Institute of Physics, The Chinese Academy of Sciences.

\section{REFERENCES}

1. J. Kressier, H. W. Kammer, and K. Klostermann, Polym. Bull., 15, 113 (1986).

2. J. S. Chiou, D. R. Paul, and J. W. Barlow, Polymer, 23, 1543 (1982).

3. K. Naito, G. E. Johnson, D. L. Allara, and T. K. Kwei, Macromolecules, 11, 1260 (1978).

4. M. E. Fowler, J. W. Barlow, and D. R. Paul, Polymer, 28, 2145 (1987).

5. T. Shiomi, M. Suzuki, M. Tohyama, and K. Imai, Macromolecules, 22, 3578 (1989).

6. J. M. E. Cowie, I. J. McEwen, and L. Nadvornik, Macromolecules, 23, 5106 (1990).

7. D. R. Paul and J. W. Barlow, Polymer, 25, 487 (1984).

8. M. Nishimoto, H. Kesskula, and D. R. Paul, Macromolecules, 23, 3633 (1990).

9. V. J. McBrierty, D. C. Douglass, and T. K. Kwei, Macromolecules, 11, 1265 (1978).

10. M. E. Fowler, J. W. Barlow, and D. R. Paul, Polymer, 28, 1177 (1987).

11. J. L. G. Pfenning, H. Keskkula, J. W. Barlow, and D. R. Paul, Macromolecules, 18, 1937 (1985).

12. R. P. Kambour, J. T. Bendler, and R. C. Bopp, Macromolecules, 16, 753 (1983).

13. G. ten Brinke, F. E. Karasz, and W. J. MacKnight, Macromolecules, 16, 1827 (1983).

14. A. C. Balasz, I. C. Sanchez, I. R. Epstein, F. E. Karasz, and W. J. MacKnight, Macromolecules, in press.

15. A. C. Balasz, F. E. Karasz, W. J. MacKnight, H. Ueda, and I. C. Sanchez, Macromolecules, 18, 2784 (1985).

16. S. E. Howe and M. M. Coleman, Macromolecules, 19, 72 (1986)

17. H. Feng, Z. Feng, and L. Shen, submitted to J. Am. Chem. Soc.

18. V. J. McBrierty and D. C. Dauglass, Phys. Rep., 63, 61 (1980).

19. A. R. Komoroski, Ed., "High Resolution NMR Spectroscopy of Synthetic Polymers in Bulk," VCH Publishers, Inc., Weinheim, Germany, 1986.

20. D. Fedotov and H. Schneider, Ed., "Structure and Dynamics of Bulk Polymers by NMR-Methods," Springer-Verlag, BerlinHeidelberg, 1989.

21. D. A. Torchia, J. Magn. Reson., 30, 613 (1978).

22. E. O. Stejskal, J. Schaefer, M. D. Sefcik, and R. A. McKay, Macromolecules, 14, 275 (1981).

23. G. A. Webb, Ed., "Annu. Rep. NMR Spectroscopy," Academic Press Inc., New York, N. Y., 1083, pp 2-10.

24. A Natansohn and A. Simmons, Macromolecules, 22, 4426 (1989).

25. H. Feng, Z. Feng, H. Yuan, and L. Shen, Macromolecules, 25, 5981 (1992).

26. H. Feng, Z. Feng, and L. Shen, in preparation. 